\title{
Structuring of Spintronic Sensors by Ion Beam Milling with in situ Insulator Deposition
}

\author{
Stefanie Rumbke ${ }^{1}$, Enrico Loos ${ }^{1}$, Matthias Nestler ${ }^{1}$, Alexander Böhnke', Niklas Dohmeier ${ }^{2}$ \\ ${ }^{1}$ scia Systems GmbH, Annaberger Straße 240, 09125 Chemnitz, Germany, \\ 2 Center for Spinelectronic Materials and Devices, Physics Department, Bielefeld University, \\ Universitätsstraße 25, 33615 Bielefeld, Germany \\ m.nestler@scia-systems.com
}

\begin{abstract}
We demonstrate that high quality $\mathrm{CoFeB} / \mathrm{MgO} / \mathrm{CoFeB}$ magnetic tunnel junctions (MTJs) can be produced by combining magnetron sputtering, UV lithography, and ion beam milling (IBM). Particularly, we study the effect of different milling angles on the tunneling magnetoresistance (TMR). Furthermore, we investigate the quality of devices manufactured by depositing the insulator in situ subsequent to milling. Using a secondary ion mass spectrometer (SIMS), all layers can be detected while milling, enabling us to precisely define the mill stops. We found TMR values of up to $140 \%$ for a milling angle of $30^{\circ}$ and $90 \%$ for a two angles milling process at $20^{\circ}$ and $65^{\circ}$, proving that no critical sidewall redeposition of conductive material takes place.
\end{abstract}

Key words: magnetic tunnel junction (MTJ), tunneling magnetoresistance (TMR), ion beam milling (IBM), dual ion beam deposition (DIBD), secondary ion mass spectroscopy (SIMS)

\section{Introduction}

In modern electronics, sensors commonly use the charge of electrons for generating a signal. However, spintronic sensors, e.g., MTJs, allow to additionally exploit the electron's spin-degree of freedom, enabling the design of versatile, inexpensive and energy efficient sensor devices on the nano-scale.

A MTJ is composed of two ferromagnetic (FM) layers with a thin non-magnetic, electrically insulating layer sandwiched between them. Electrons can tunnel through this barrier from one FM layer to the other, with their spin conserved. The upper FM layer is soft magnetic, i.e., its magnetization can easily be changed by applying an external magnetic field. It is thus called the free layer. The lower FM layer is, in contrast, hard magnetic and called the pinned layer, i.e., a stronger magnetic field is required to change its magnetization than in the case of the free layer. A reversal of the relative magnetic orientation of these two layers can thus be accomplished by applying an external field that is strong enough to change the magnetization of the free layer, but too weak to affect the pinned layer. This induces a change in electric resistivity, caused by a different probability for electrons to tunnel through the tunnel barrier. The tunneling probability is higher for a parallel $(P)$ magnetization of the FM layers and lower for an antiparallel (AP) magnetization. Accordingly, the TMR is higher for the AP than for the $P$ magnetic orientation, an effect first observed in 1975 [1]. The magnitude of the TMR is expressed by the magnetoresistance ratio

$\frac{\Delta R}{R}=\frac{R_{\mathrm{AP}}-R_{\mathrm{P}}}{R_{\mathrm{P}}}$

where $R_{\mathrm{AP}}$ and $R_{\mathrm{P}}$ are the respective resistances for the $A P$ and $P$ magnetic states $[2,3]$. Today, MTJs based on $\mathrm{CoFeB} / \mathrm{MgO} /$ CoFeB form the backbone of high quality spintronic devices. Records for the TMR lie at $604 \%$ at room temperature and $1144 \%$ at $5 \mathrm{~K}$ for these materials [4].

\section{Experimental}

The magnetic stacks used in this study are composed of various layers deposited by magnetron sputtering on a Si substrate with a SiO layer formed on top (see Fig. 1). An underlayer consisting of $5 \mathrm{~nm} \mathrm{Ta}, 30 \mathrm{~nm} \mathrm{Ru}$ and again $10 \mathrm{~nm} \mathrm{Ta}$ and $10 \mathrm{~nm} \mathrm{Ru}$ is deposited first, followed by an antiferromagnetic layer of $20 \mathrm{~nm} \mathrm{Mnlr}$. This layer is used to exchangebias the pinned layer, consisting of $3 \mathrm{~nm}$ CoFe. This, combined with $0.8 \mathrm{~nm} \mathrm{Ru}$ and $3 \mathrm{~nm}$ CoFeB, forms an artificial antiferromagnet, followed by the tunneling barrier, composed of $1.7 \mathrm{~nm} \mathrm{MgO}$, and the free layer, composed of 3 $\mathrm{nm}$ CoFeB. Finally, a cap layer of $5 \mathrm{~nm}$ Ta and 
$5 \mathrm{~nm} \mathrm{Ru}$ is deposited on the stack. The samples are annealed at $360{ }^{\circ} \mathrm{C}$ for $60 \mathrm{~min}$ in an external field of about 5500 Oe. By this process step, pinning is induced and the $\mathrm{MgO}$ layer is crystallized. The completed stack is then patterned by masks made of positive photoresist for the formation of the upper contacts. The unmasked material is removed by IBM, i.e., it is removed by bombardment with a neutralized ion beam provided by a broad ion source that is directed to the substrate, using $\mathrm{Ar}$ as a process gas. To investigate the influence of different milling angles, some stacks are milled at an angle of incidence of $30^{\circ}$ perpendicular to the sample surface (see Fig. 1 (a)) and others at two different angles (see Fig. 1 (b)). The first layers are milled at a steeper angle of $20^{\circ}$ around the mask, until the $\mathrm{MgO}$ tunnel barrier is reached. All following layers are removed under a shallower angle of $65^{\circ}$ in order to avoid redeposition of conductive material at the insulating barrier. At the same time, the ion beam cleans the lateral faces of the layers. The milling process is controlled by SIMS to detect the layer boundaries and determine the mill stop for the lower contact as well as the changing point for the angle in (b) (see Fig. 2). The spectrometer is sensitive to layer thicknesses in the single-digit $\mathrm{nm}$ range, making it possible to detect all layers in the stack and thus precisely define the end points. Prior to contacting the layers, they need to be encapsulated by an insulator. For this purpose,

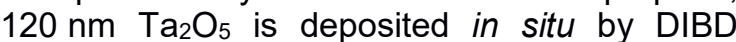
using a metallic Ta target and additionally added oxygen as a reactive background gas. The ion beam provided by the sputter source is focused on the target, sputtering the material that is to be deposited on the substrate. The assist source (the ion source formerly used to remove the unmasked layer stack) is here used to improve the properties of the deposited layer.

\section{Results}

A major (left) and a minor hysteresis loop (right) are shown in Fig. 3 for MTJs milled at $30^{\circ}$ (Fig. $3(\mathrm{a})$ ) and $20^{\circ} / 65^{\circ}$ (Fig. 3 (b)). The graphs show a TMR value of $140 \%$ for $30^{\circ}$ and $90 \%$ for $20 \% 65^{\circ}$. If the range of the applied magnetic field is high enough, both the exchange biased pinned layer and the free layer can be switched (major loop). The TMR values decrease with the applied field in both directions, with a sharp drop for the switching of the free layer and a smoother decrease for the pinned layer. By choosing a more limited field range, only the magnetization of the free layer can be changed whereas that of the pinned layer cannot (minor loop). A steep fall of the TMR value can be observed for $30^{\circ}$ while it drops at a shallower angle for $20^{\circ} / 65^{\circ}$.

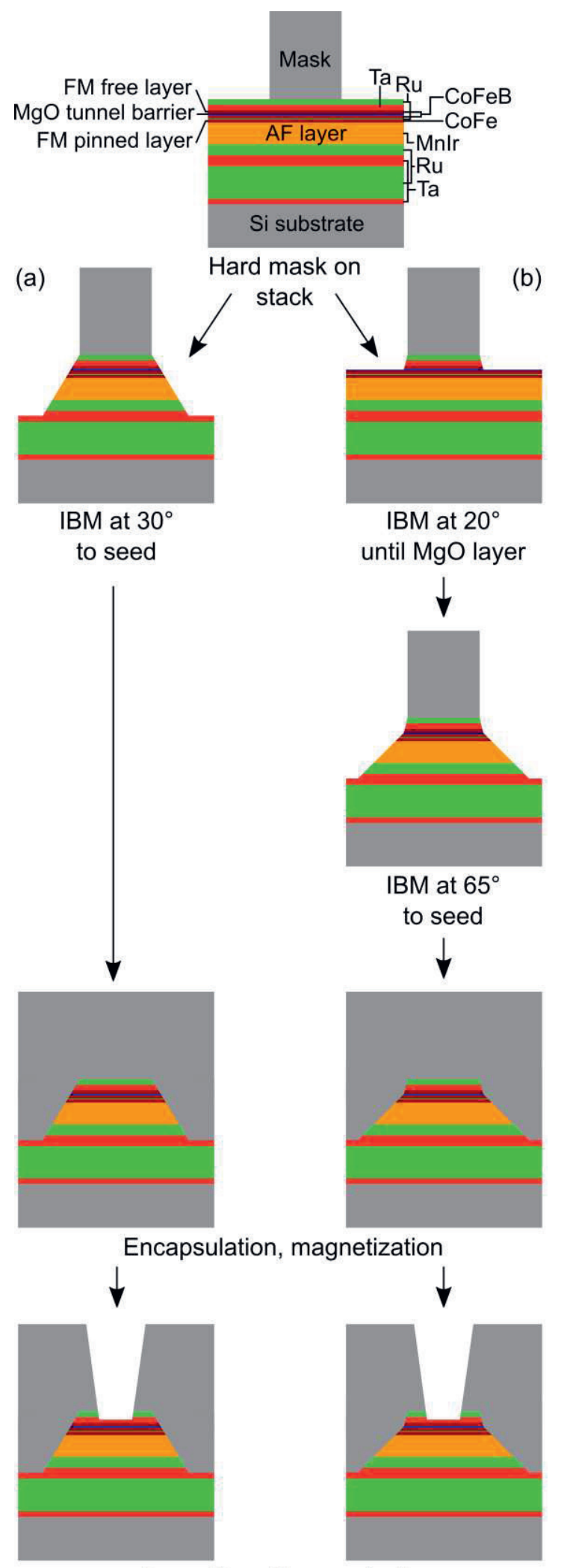

Formation of top contact

Fig. 1. Sketch of the cross-section of the $\mathrm{CoFeB} / \mathrm{MgO} / \mathrm{CoFeB} \mathrm{MTJ}$ showing the manufacturing process. After depositing all layers and the masks on the stack, it is milled down to the seed, encapsulated with $\mathrm{Ta}_{2} \mathrm{O}_{5}$, and magnetized. In a final step, the top contact is formed. Milling is either performed (a) at an angle of $30^{\circ}$ or (b) at $20^{\circ}$ to the $\mathrm{MgO}$ tunneling barrier and then at $65^{\circ}$ down to the seed. 


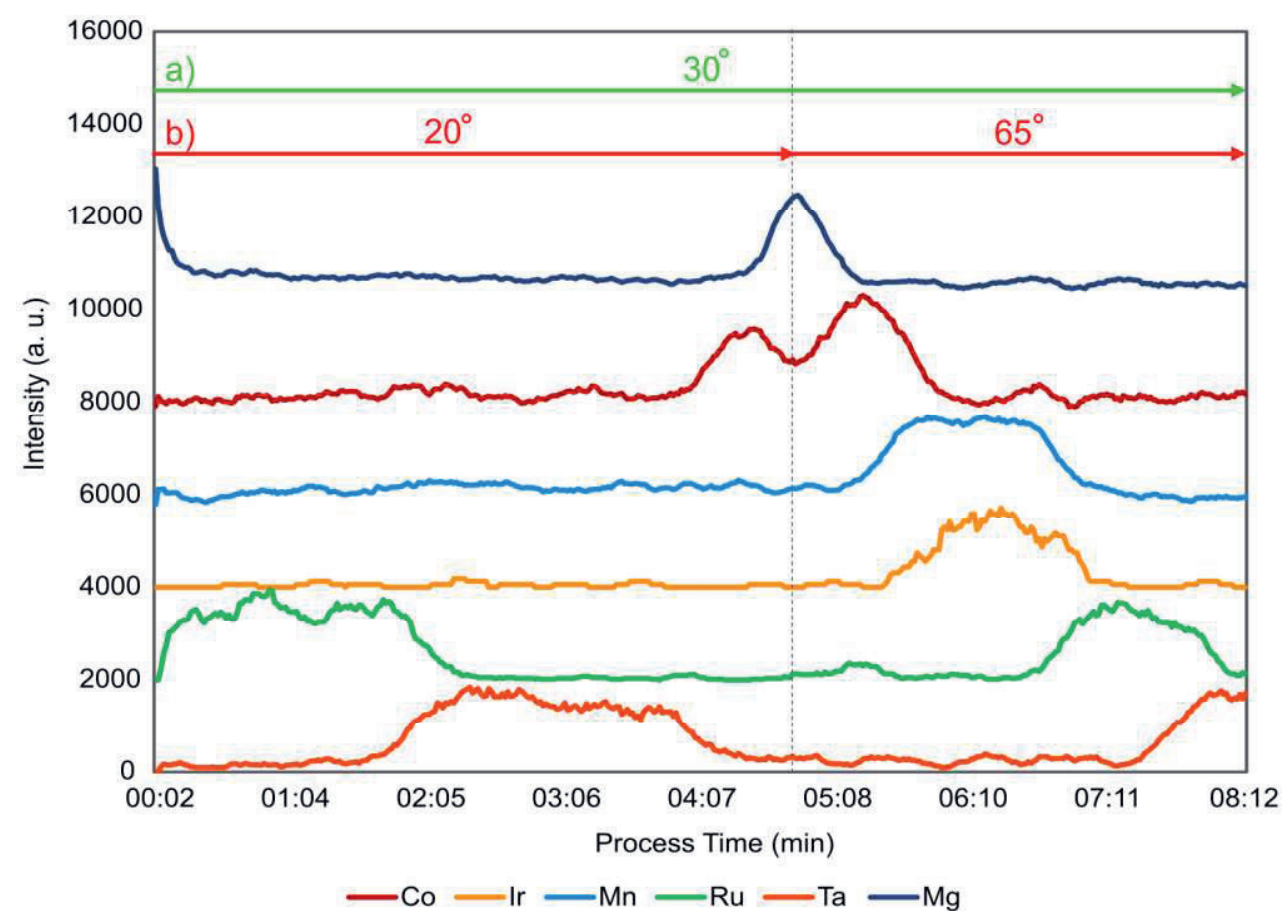

Fig.2. Signal for different materials present in the layer stack detected by the Secondary lon Mass Spectrometer. With this method, the layer boundaries and thus the mill stops can be determined. (a) The stack is milled down to the $10 \mathrm{~nm}$ Ta layer. (b) The stack is milled down to the $1.7 \mathrm{~nm} \mathrm{MgO}$ tunneling barrier, then the angle is adjusted and the milling is continued until the $10 \mathrm{~nm}$ Ta layer is reached.

a)

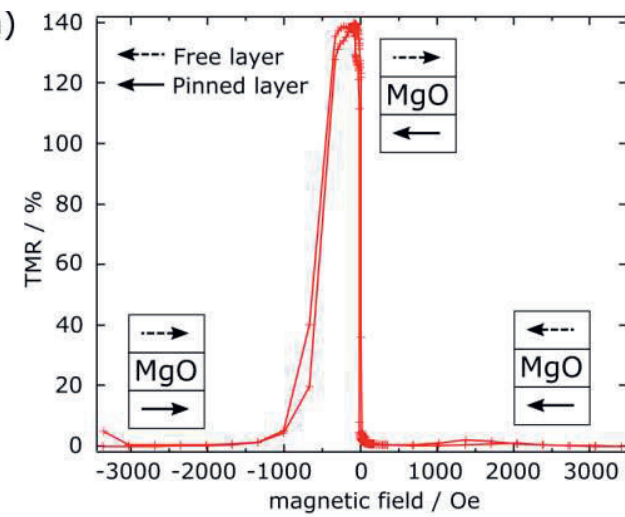

b)

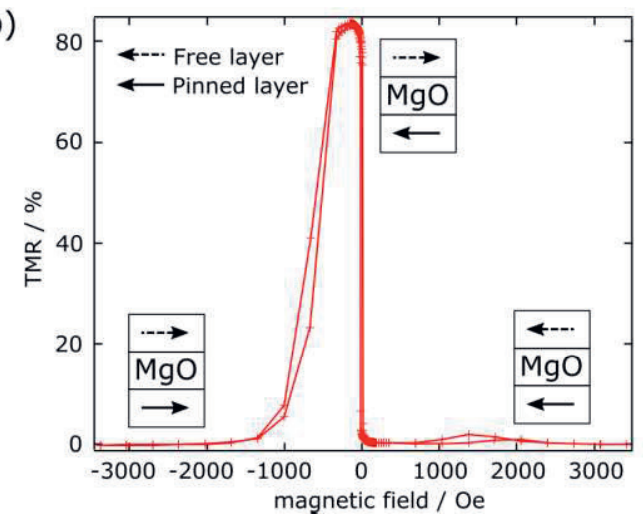

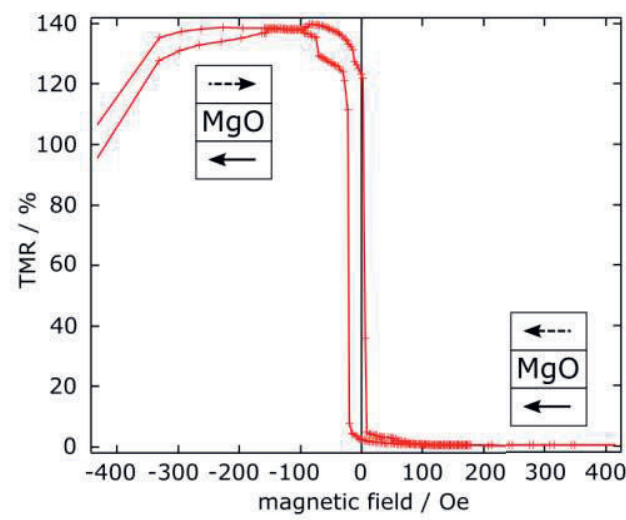

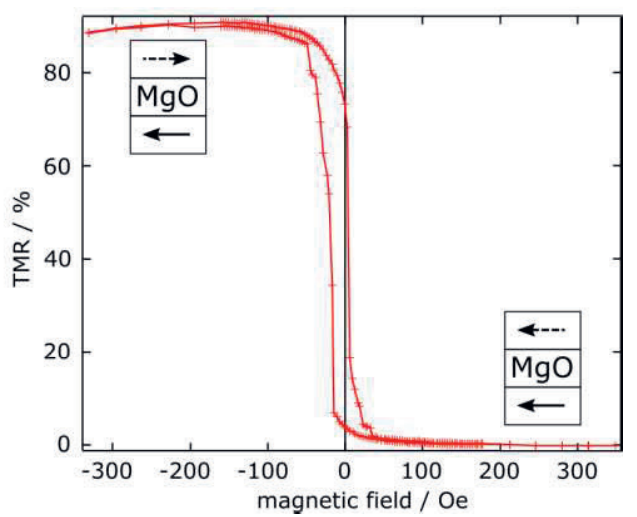

Fig.3. TMR plotted versus the applied magnetic field. By selecting a high enough field range, both the exchange biased pinned layer and the free layer can be switched (left). In a reduced field range, only the pinned layer changes its magnetization (right). (a) Layer stack milled at $30^{\circ}$. (b) Layer stack milled at $20^{\circ}$ and $65^{\circ}$. 
The shallow angle of $65^{\circ}$ in (b) was chosen deliberately in order to avoid bypasses by redeposition of metal at the $\mathrm{MgO}$ barrier. The TMR measurements show, however, high values for the $30^{\circ}$ sample as well, indicating a lack of critical sidewall deposition also for this angle. The shallower drop of the TMR value compared to the $30^{\circ}$ device may indicate a rougher free layer supporting the lower TMR value for this sample, making a statement about the angle dependency of the TMR difficult. This needs to be clarified in a follow-up experiment, in which also the geometry of the device edges could be examined by e.g. SEM.

\section{Summary}

We demonstrated that high TMR values can be obtained for Co-Fe-B/MgO/Co-Fe-B MTJs by depositing the insulator in situ subsequent to milling. Using a SIMS, all layers can be detected while milling, so that all mill stops can precisely be defined. A TMR value of $140 \%$ could be measured for a milling angle of $30^{\circ}$ and $90 \%$ for the two steps milling process with $20^{\circ} / 65^{\circ}$. There is evidence that both processes lead to functional devices without a critical sidewall redeposition of conducting material that would decrease the TMR signal. The reproducibility of the results and if there is a real angle dependency has yet to be investigated.

\section{References}

[1] M. Julliere, Tunneling Between Ferromagnetic Films, Physics Letters A 54, 225-226 (1975); doi: 10.1016/0375-9601(75)90174-7

[2] J. S. Moodera, L. R. Kinder, T. M. Wong, R. Meservey, Large Magnetoresistance at Room Temperature in Ferromagnetic Thin Film Tunnel Junctions, Physical Review Letters 74, 32733276 (1995), doi: 10.1103/PhysRevLett.74.3273

[3] T. Miyazaki, N. Tezuka, Spin polarized tunneling in ferromagnet/insulator/ferromagnet junctions, Journal of Magnetism and Magnetic Materials 151, 403-410 (1995), doi: 10.1016/03048853(95)00563-3

[4] S. Ikeda, J. Hayakawa, Y. Ashizawa, Y. M. Lee, K. Miura, H. Hasegawa, M. Tsunoda, F. Matsukura, $\mathrm{H}$. Ohno, Tunnel magnetoresistance of $604 \%$ at $300 \mathrm{~K}$ by suppression of Ta diffusion in $\mathrm{CoFeB} / \mathrm{MgO} / \mathrm{CoFeB}$ pseudo-spin-valves annealed at high temperature, Applied Physics Letters 93, 082508 (2008); doi: 10.1063/1.2976435 